\title{
Investigation of time dependent inner core structure by the analysis of free oscillation spectra
}

\author{
D. S. Sharrock and J. H. Woodhouse \\ Department of Earth Sciences, Oxford University, Parks Rd., Oxford OXI 3PR, U.K.
}

(Received April 20, 1998; Revised August 10, 1998; Accepted August 11, 1998)

\begin{abstract}
Proposals that the seismic structure of the Earth's inner core is time-dependent are investigated using the splitting functions of free oscillations, determined from seismic data recorded over some 20 years. It is shown that the data support some time-dependence, although the paucity of the data for the 1970's and 1980's makes it difficult to be certain of the precise form of such time dependence. The data do not support a positive (i.e. West-to-East) rotation; rather, the inferred rotational component of the time-dependent inner core structure is negative (i.e. East-to-West), and varies according to the mode under consideration from less than $1^{\circ}$ per year to more than $2^{\circ}$ per year. The analysis suggests that inner core structure is time-dependent but that the dependence is not well explained by differential rotation of the inner core. This kind of analysis will become much more powerful with increasing quantities of data over a longer time span.
\end{abstract}

\section{Introduction}

Several investigations using body wave seismograms and body wave arrival times (Song and Richards, 1996; Su et al., 1996; Creager, 1997) have concluded that there is evidence of time-dependence of inner core structure. Such time-dependence has been ascribed to differential rotation of the inner core at rates varying from approximately $1^{\circ}$ per year (Song and Richards, 1996), $3^{\circ}$ per year (Su et al., 1996) to a small fraction of a degree per year (Creager, 1997), in each case from West to East.

The interpretation of time dependence in terms of inner core differential rotation has been motivated by recent results from magnetohydrodynamic numerical models in which differential rotation occurs at the rate of a few degrees per year in the W to E sense (Glatzmaier and Roberts, 1995) or with fluctuating sense (Kuang and Bloxham, 1997). The potential to discriminate between different modes of dynamo action makes the study of time dependent phenomena in the inner core of great interest. There is also the possibility that seismic observations will require more general time dependence and thus provide totally new information on core dynamics.

The evidence for time-dependence of Song and Richards (1996) has been disputed (Souriau et al., 1997). If time dependence is accepted, however, their inference that it is due to inner core rotation, and the estimation of a rotation rate, remain problematic. Assuming that differential rotation is the cause, the observed travel time variations are ascribed to the rotation of the aspherical (by which we shall mean any structure deviating from spherical symmetry) inner core model of Su and Dziewonski (1995). This model is an approximation to inner core structure, representing only the longest wavelengths of asphericity. Any shorter wavelength structure su-

Copy right(C) The Society of Geomagnetism and Earth, Planetary and Space Sciences (SGEPSS); The Seismological Society of Japan; The Volcanological Society of Japan; The Geodetic Society of Japan; The Japanese Society for Planetary Sciences. perimposed on the model could greatly alter the predicted travel time difference associated with a given rotation angle, and since such shorter wavelength structure cannot be ruled out there is little reason to put faith in the estimated rotation rate of approximately $1^{\circ}$ per year. Indeed, Creager (1997) has argued that there are strong lateral gradients in inner core velocities and his estimates of rotation rate are much smaller ( 0.05 to $0.3^{\circ}$ per year). Because the number of ray paths is small it is possible that the time dependence is not associated with rotation at all but, rather, with some other phenomenon, yet to be determined - for example, mobile topographic features at the inner core boundary ("sludge"), possibly in combination with differential rotation of undetermined sign and magnitude.

Su et al. (1996) make use of the PKIKP arrival times reported in the Bulletin of the International Seismological Centre. This constitutes a vastly more numerous data set, spanning some 30 years, in principle capable of imaging the inner core through time and thus determining whether rotation is the cause of time-dependence. Su et al. (1996) determine a rotation rate of some $3^{\circ}$ per year. It seems possible, however, that the data could also support a more complex kind of time dependence. While numerous, ISC data are "noisy" and suffer from time-dependence caused by the varying complement of stations reporting and changes in seismic reading practices and in station personnel at the reporting stations.

In all such studies it is also necessary to cope with trends in the distribution of earthquakes over the interval of study. For example, the earthquakes studied by Creager (1997) exhibit a general migration across the zone of interest, making it more difficult to be certain the observed time dependence can be uniquely associated with inner core phenomena.

Free oscillation splitting functions (Woodhouse and Giardini, 1985) provide a quite different method of investigating inner core structure and its time dependence. Observations of anomalous splitting in free oscillation spectra 


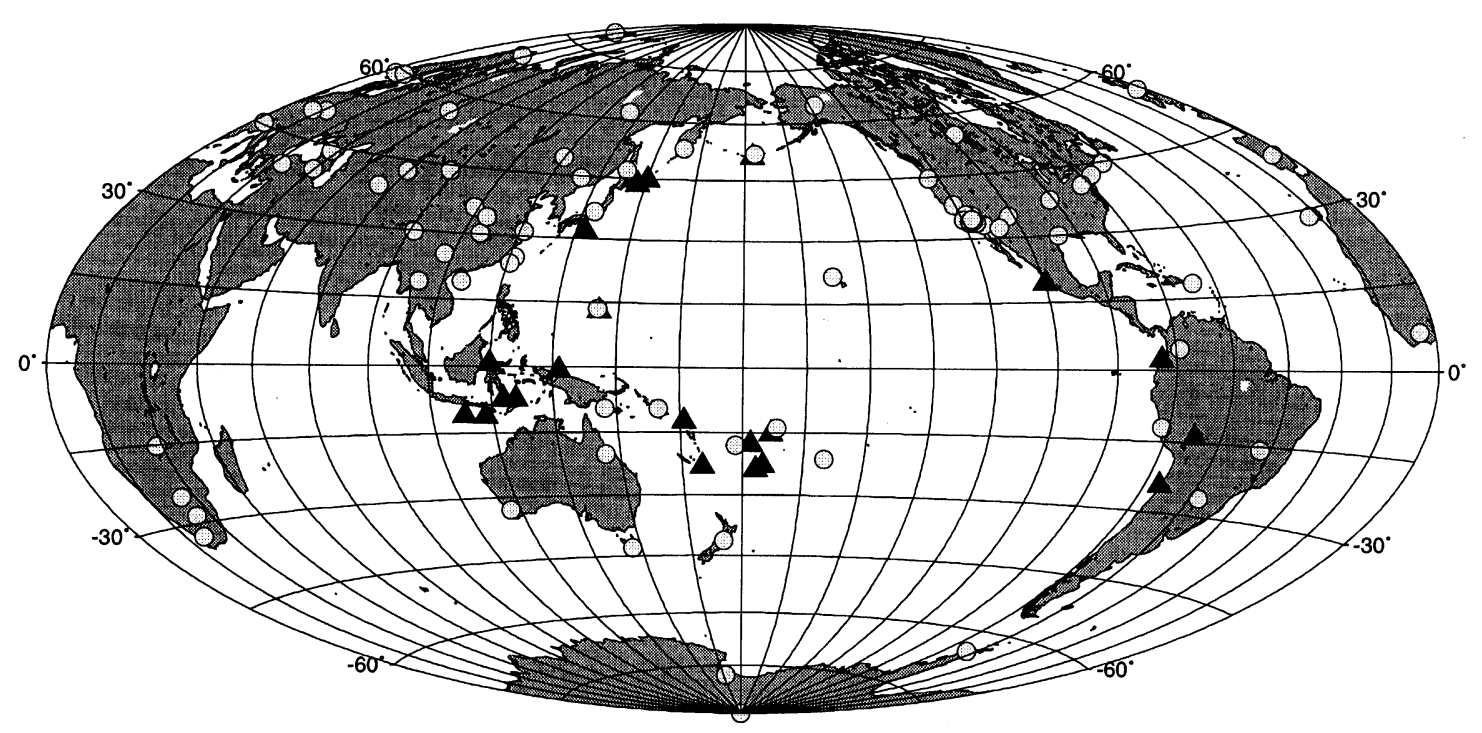

Fig. 1. Map showing the distribution of earthquakes (triangles) and stations (circles) used in this study.

(Ritzwoller et al., 1986), analysed by the splitting function or spectral fitting technique (Woodhouse et al., 1986) led, in conjunction with a companion paper on travel times (Morelli et al., 1986), to the discovery of zonal anisotropy of the inner core, confirmed in a number of subsequent studies (for a review see Song, 1997). This, the largest long wavelength structure in the inner core, is symmetric under rotations about the Earth's rotation axis, and does not contribute to time variations in seismic properties caused by differential rotation. It is the nonzonal component of inner core structure that is of interest for the study of inner core rotation. Inner core mode splitting functions are not entirely zonal, even when account is taken of mantle heterogeneity, and thus the investigation of the time dependence of splitting functions offers an alternative approach to the study of inner core time dependence and rotation. Potentially this approach has great advantages over body wave studies, in that splitting functions represent a global image (averaged over radius according to the sensitivity kernels of each particular mode) and therefore have the potential to discriminate between rotation and more general kinds of time-dependence. If the inner core is differentially rotating one should observe that the splitting functions of inner core modes, corrected for mantle structure, and assuming no outer core heterogeneity (see Stevenson, 1987), all display a similar amount of rotation over a given time interval; this would be proof of inner core rotation. The potential problems are associated (i) with our ability to correct adequately for mantle structure and (ii) with the fact that data suitable for such studies are sparse before the 1990's. The large, deep earthquakes that are necessary to excite inner core modes are rare, and there were relatively few suitable seismometers deployed before the 1990's.

In this study we analyse the data for some of the better observed inner core modes in order to assess the evidence for time dependence and rotation. In Section 3 we address the question of the compatibility of the data sets for the two different epochs for a number of modes that both sample and do not sample the inner core. It is found that some time dependence of inner core structure is indicated, and the kind of time dependence required is illustrated with the mantle-corrected splitting function of ${ }_{3} S_{2}$, a mode that is characterised by high sensitivities to inner core anisotropy and heterogeneity. In Section 4 we analyse a number of inner core modes under the assumption that there is inner core rotation at a specified rate. By varying the assumed rate we seek the optimal rotation rate for each mode.

\section{Data Processing}

We use data from the Global Digital Seismic Network and International Deployment of Accelerometers recording a suite of earthquakes spanning nearly two decades. Figure 1 shows the distribution of earthquakes and stations used in this study. The more recent earthquakes are recorded by more than 60 stations worldwide. Each record is visually inspected and any glitches, clipped segments and excessively noisy segments are removed. The tidal signal is also removed. Any record shorter than 30 hours is discarded. The data are windowed using a Hann taper, padded with zeros, and the instrument response removed to leave a record of ground acceleration.

We adjust the start time and length of the unpadded time series before applying a Fourier transform to give the highest signal-to-noise ratio in the frequency domain. A long start time delay can be useful for observing high- $Q$ modes which are often swamped by low- $Q$ mantle modes. A long record can be useful for observing more detailed splitting characteristics, particularly for quiet records. We calculate synthetic seismograms using the source mechanism and epicentre location given by the Harvard Centroid Moment Tensor (CMT) analysis. The locations and depths of the earthquakes used are given in Table 1. The synthetic seismograms are then processed in exactly the same way as the data.

The spectral splitting of the Earth's normal modes departs from the splitting predicted on the basis of rotation 
Table 1. Date and location of the earthquakes used in this study. The dotted line distinguishes between the two time periods mentioned in the text.

\begin{tabular}{|c|c|c|c|c|}
\hline Date & Event & Latitude & Longitude & Depth $(\mathrm{km})$ \\
\hline 06/17/96 & Flores Sea & -7.38 & 123.02 & 584.2 \\
\hline $06 / 10 / 96$ & Andreanof Islands & 51.10 & -177.41 & 29.0 \\
\hline $02 / 17 / 96$ & West Irian Region & -0.67 & 136.62 & 15.0 \\
\hline $01 / 01 / 96$ & Minahassa Peninsula & 0.74 & 119.93 & 15.0 \\
\hline $12 / 03 / 95$ & Kurile Islands & 44.82 & 150.17 & 25.9 \\
\hline $10 / 09 / 95$ & Near Coast of Jalisco & 19.34 & -104.80 & 15.0 \\
\hline $07 / 30 / 95$ & Near Coast of N Chile & -24.17 & -70.74 & 28.7 \\
\hline $05 / 16 / 95$ & Loyalty Islands & -23.05 & 170.00 & 24.7 \\
\hline $04 / 07 / 95$ & Tonga Islands & -15.37 & -173.15 & 87.9 \\
\hline $10 / 04 / 94$ & Kurile Islands & 43.60 & 147.63 & 68.2 \\
\hline 06/02/94 & South of Java & -11.03 & 113.04 & 15.0 \\
\hline 06/09/94 & Northern Bolivia & -13.82 & -67.25 & 647.1 \\
\hline 03/09/94 & Fiji Islands & -17.69 & -178.11 & 567.8 \\
\hline $08 / 08 / 93$ & South of Mariana Islands & 13.06 & 145.31 & 59.3 \\
\hline $06 / 22 / 82$ & Banda Sea & -7.28 & 125.99 & 473.4 \\
\hline $07 / 17 / 80$ & Santa Cruz Islands & -12.44 & 165.94 & 34.0 \\
\hline $04 / 13 / 80$ & Tonga Islands & -23.74 & -176.70 & 166.2 \\
\hline $12 / 12 / 79$ & Near Coast of Ecuador & 2.32 & -78.81 & 19.7 \\
\hline $12 / 06 / 78$ & Kurile Islands & 44.74 & 145.82 & 181.0 \\
\hline $03 / 07 / 78$ & South of Honshu & 31.90 & 137.44 & 434.4 \\
\hline 08/19/77 & South of Sumbawa Island & -11.14 & 118.23 & 23.3 \\
\hline $06 / 22 / 77$ & Tonga Islands & -22.86 & -174.91 & 61.3 \\
\hline
\end{tabular}

and ellipticity because of lateral heterogeneity in the structure of the Earth's core and mantle and because of deviations from hydrostatic ellipticity of the major internal discontinuities. The splitting of a normal mode is given by the splitting coefficients - the spherical harmonic expansion coefficients of the splitting function-which are linearly related to three-dimensional (3-D) structure (Woodhouse and Giardini, 1985). We invert for the splitting coefficients using an initial model incorporating the known effects of rotation and ellipticity, and a model of mantle structure. We use the degree-12 shear velocity model (S12WM13) of Su et al. (1994). Thus we invert for deviations from a 3-D mantle model, which we assign to the effects of core heterogeneity. The technique is essentially that described by Giardini et al. $(1987,1988)$. Splitting functions are determined up to degree and order 4.

\section{Time Dependence in Normal Mode Observa- tions}

We investigate time-varying inner core structure using inner core modes observed over a twenty year period. The large number of stations recording the recent large deep earthquakes (Table 1) result in well constrained models using more than 70 mode observations in most cases. In the 1970's and
80 's the station coverage was not as good and there are fewer observations of the large deep earthquakes from this period. In order to investigate time-dependence we select two suites of data. The first spans the six years 1977-1982 and will be referred to as D77/82; the second spans four the four years 1993-1996 and will be referred to as D93/96. Table 2 gives the number of mode observations for each dataset.

In principle, to establish time dependence we need simply to invert each data suite and compare the results. In practice, error and instability in the inversions need to be taken into account, particularly for those of the earlier period. We need to ask such questions as whether there are time-independent solutions that adequately fit both suites of data, or whether the data suites are inconsistent. If the data suites are consistent (i.e. there is no time dependence) and sufficient to determine the splitting coefficients we would expect to obtain the same result for all combinations of the data. In the presence of uncertainties in the solutions corresponding to one or the other data suite, it should nevertheless be the case that there exist solutions providing an optimal fit to both suites.

To investigate this we define a sequence of inversions depending on a parameter $\eta$ which defines the relative weight with which the two data suites enter into the inversion. This 
Table 2. Each mode is listed along with the number of observations in each of the three time periods. Modes sensitive only to the mantle and outer core are shown by an asterisk.

\begin{tabular}{cccc}
\hline \multirow{2}{*}{$\begin{array}{c}\text { Observed } \\
\text { mode }\end{array}$} & \multicolumn{3}{c}{ Number of observations } \\
\cline { 2 - 4 } & $1977-96$ & $1977-82$ & $1993-96$ \\
{$[\mathrm{D} 77 / 82]$} & {$[\mathrm{D} 93 / 96]$} \\
\hline${ }_{3} S_{2}$ & 57 & 16 & 41 \\
${ }_{9} S_{3}$ & 94 & 15 & 79 \\
${ }_{11} S_{4}$ & 149 & 21 & 128 \\
${ }_{11} S_{5}$ & 114 & 16 & 98 \\
${ }_{13} S_{3}$ & 85 & 13 & 72 \\
${ }_{0} S_{13}^{*}$ & 336 & 34 & 302 \\
${ }_{5} S_{6}^{*}$ & 154 & 20 & 134 \\
\hline
\end{tabular}

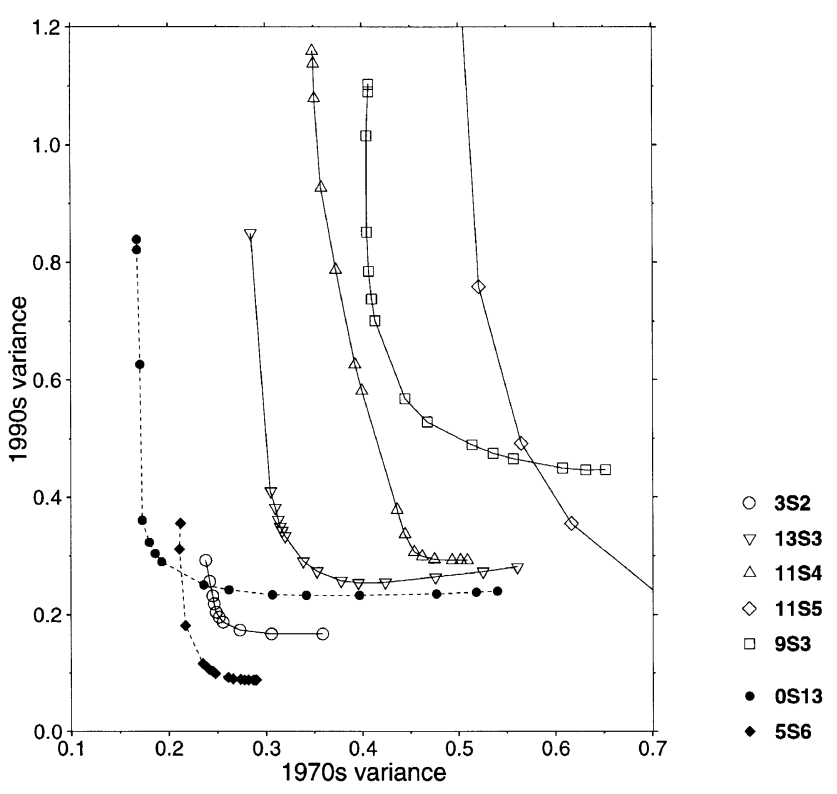

Fig. 2. Absolute variance for D77/82 versus D93/96 for a range of models calculated using different combinations of data. The dotted lines are mantle modes and the solid lines are inner core modes. See legend for individual modes. The inner core mode ${ }_{11} S_{5}$ shows a larger range in variance. The asymptotic $1970 \mathrm{~s}$ variance value is 0.454 , and the asymptotic 1990s variance is 0.171. An L-shaped variance curve defines combined data models derived from consistent datasets (see text for complete description).

is defined in such a way that $\eta=1$ corresponds to inversion only for $\mathrm{D} 77 / 82$ and $\eta=0$ corresponds to inversion only for D93/96. We then examine the behaviour of the misfit variance ratios of the two data sets (squared misfit/squared data). A plot of the variance ratio of one data set against the other can be used to assess the consistency of the data sets. In such a plot (see below) we would expect that if the data suites are consistent there will be values of $\eta$ that lead to solutions that have variance ratios for both data suites that are close to the optimum for each data suite on its own. This leads to a plot resembling the letter " $L$ " in which the extent of
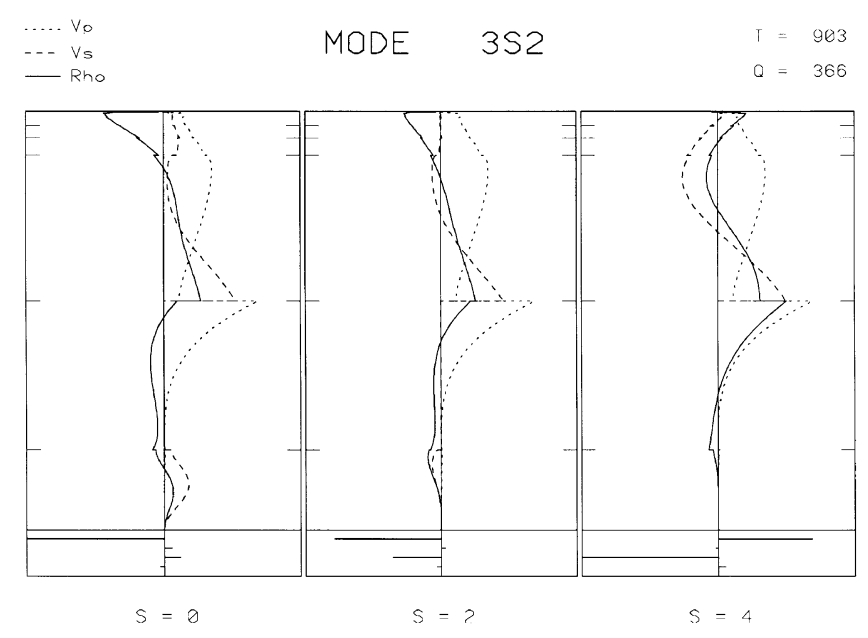

Fig. 3. The sensitivity kernel for ${ }_{3} S_{2}$. The kernels represent the sensitivity, as a function of depth, of the splitting function elements to a $1 \%$ perturbation in $v_{p}$ (dotted line), $v_{s}$ (dashed line) and $\rho$ (solid line). For a given mode the kernels are independent of angular order $t$, and only weakly dependent on degree $s$. Here we show separate kernels for degree $s=0,2,4$ and for $v_{p}, v_{s}$ and $\rho$ centred on the zero line.

the two limbs of the "L" represent the failure of the solution derived from one data suite to optimally fit the other suitethe result of instabilities in the solution derived from one data suite on its own. The solution corresponding to the corner of the " $L$ " represents a solution that provides an optimal fit to both data suites. On the other hand, if the data suites are inconsistent, intermediate values of $\eta$ lead to solutions that represent a compromise between the data suites, and so we would find that the plot is less angular, indicating that there is no solution representing an optimal fit to both data suites.

Figure 2 shows such variance ratio plots for a number of modes. Modes ${ }_{0} S_{13}$ and ${ }_{5} S_{6}$ (solid symbols) do not sample the inner core, and are closest to the ideal "L" shape. On the other hand, for the inner core modes the variance curve deviates from the L-shape. This deviation is a result of inconsistencies in the datasets: we cannot find a combined data model which fits the data as well as the D93/96 data alone. This inconsistency is evidence of time-dependent structure.

To illustrate the kind of time dependence that may be required we consider the inner core mode ${ }_{3} S_{2}$. The sensitivity kernel for ${ }_{3} S_{2}$ is shown in Fig. 3 and Fig. 4 shows the familiar strongly zonal pattern of the splitting function for ${ }_{3} S_{2}$. The mantle shear model has been removed so that the splitting function shown represents deviations from a rotating, elliptical Earth with a given shear velocity structure in the mantle. Hence, the splitting functions can be interpreted in terms of core structure.

Some of the features in the D77/82 model (Fig. 4(a)), such as the low velocity spurs along the west coast of North America in the northeast quadrant and southeast of the tip of Africa in the southwest quadrant, are still present in the D93/96 model (Fig. 4(c)), although less pronounced; more of a saddle feature in the strongly zonal pattern. Removing the zonal terms from the splitting function (Fig. 5) brings these features to the fore. The amplitude of the spur-like features is lower in the most recent model (Fig. 5(c)), although 

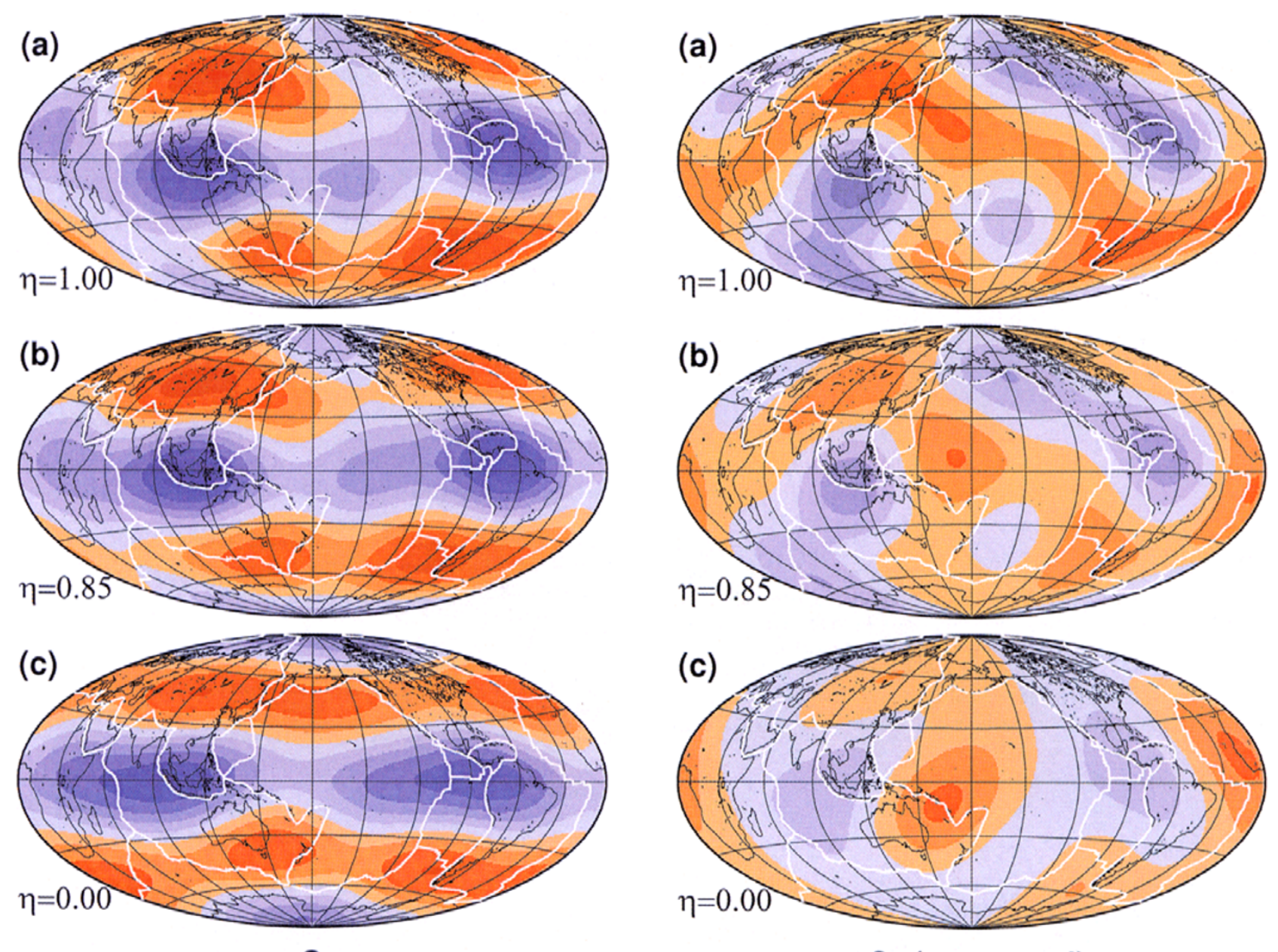

${ }_{3} \mathrm{~S}_{2}$

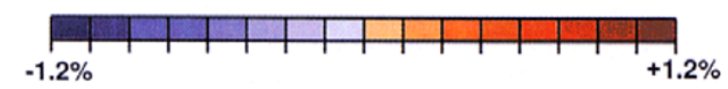

Fig. 4. Three splitting functions for the $\operatorname{mode}_{3} S_{2}$, for $\eta=1.0,0.85$ and 0.0 . Only even degrees of the real harmonic expansion of the splitting function are displayed. The maximum scale is $1.2 \%$ of the mode frequency.

the geographical location of the spurs is approximately unchanged. There is also a small translation of the main body of the spur to the west, from the D77/82 model to the D93/96 model, most noticeable in the feature located near Sumatra (northwest of Australia). This may be a result of a westward differential rotation of the inner core. It should be noted though, that the principal change cannot be associated with rotation.

\section{Seeking the Rate of Differential Rotation}

Differential rotation has been considered the most likely mechanism for time-dependent observations relating to the core. Here we describe experiments in the inversion of the whole data set under the assumption that differential inner core rotation occurs at a specified rate, varying from -5 to $+5^{\circ}$ per year. We iteratively solve the inverse problem for a range of rotation rates. In Fig. 6 we present the results for four inner core modes for which stable solutions were found. All four modes show a minimum in misfit variance ratio, which in all cases indicates a westward (negative) rotation. The minimum absolute variance is clearly identified for three of the four modes $\left({ }_{13} S_{3}\right.$ appears to be noisier than the other modes) and represents a change of a couple of percent in variance reduction. The data indicate rotation rates less than $2.5^{\circ}$ per year westward.

A measure of the level of variance fluctuation that should be considered significant can be obtained assuming that $\sigma^{2} / \sigma_{\min }^{2}$ is distributed as $\chi^{2} / N$, where $\sigma^{2}$ denotes the variance ratio plotted in Fig. 6 , and $N$ is the number of degrees of freedom. The value of $N$ can be estimated as in Giardini et al. (1988); the values of $N$ for each mode are detailed in the figure caption to Fig. 6 . Using the asymptotic properties of the $\chi^{2}$ distribution (see e.g. Abramowitz and Stegun, $1965)$ we find $\sigma^{2} / \sigma_{\min }^{2}<1+1.81 / \sqrt{N}$ with $90 \%$ confidence. These variance thresholds are indicated in Fig. 6. It will be noted that in all cases the region below the threshold spans the dashed line, representing $0^{\circ}$ per year rotation, indicating that rotation does not significantly reduce the vari- 


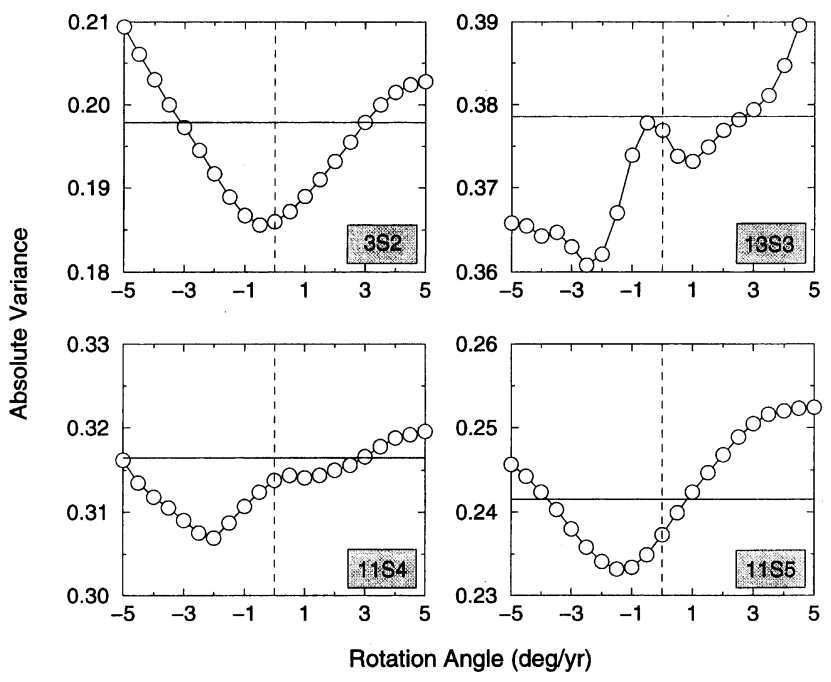

Fig. 6. Graphs of absolute variance as a function of rotation rate. Most of the inner core modes show a preference in rotation rate, typically a small rotation rate in the westward direction, opposite to that proposed by the PKP studies. The mode is given at bottom right of each graph, and zero rotation rate is shown by the vertical dashed line. The $90 \%$ confidence threshold is shown by the horizontal solid line. The number of degrees of freedom associated with each mode are as follows: ${ }_{3} S_{2}(684) ;{ }_{13} S_{3}$ (1241); ${ }_{11} S_{4}$ (3456); ${ }_{11} S_{5}$ (2459).

ance. (These thresholds should be considered only a guide to the significant levels of variance fluctuation as they depend upon the simplifying assumption of independent normally distributed errors.)

If differential rotation is the only source of time-dependent structure on this time scale we expect to be able to convert a variance curve such as those shown in Fig. 2 for inconsistent data to a more L-shaped curve indicative of consistent data. In fact, we do not find this. The indicated rotation rates have little or no effect on the variance curve from which we conclude that there are other kinds of time variation having an effect on time scales of the order of tens of years.

\section{Conclusions}

We have shown evidence of differences or inconsistencies between two modal datasets separated by 10 years, which can be interpreted as an indication of time-dependent inner core structure. We model this variation in terms of differential rotation of the inner core with respect to the overlying mantle. Although we deduce a preferred rotation rate for a number of inner core modes, rotation does not remove the inconsistency between datasets. While there appears to be time-dependent structure in the inner core, as shown by both differential travel times and the normal mode observations discussed here, it cannot be attributed purely to differential rotation, and other possible mechanisms for inner core time variation should be considered.

Acknowledgments. We thank Guy Masters and an anonymous reviewer for constructive comments which have improved this paper. This research was funded by NERC under fellowship GT5/95/ES/9.

\section{References}

Abramowitz, M. and I. A. Stegun, Handbook of Mathematical Functions, Dover Publications, New York, 1965.

Creager, K. C., Inner core rotation rate from small-scale heterogeneity and time-varying travel times, Science, 278, 1284-1288, 1997.

Giardini, D., X.-D. Li, and J. H. Woodhouse, Three-dimensional structure of the Earth from splitting in free oscillation spectra, Nature, 325, 405-411, 1987.

Giardini, D., X.-D. Li, and J. H. Woodhouse, Splitting functions of longperiod normal modes of the Earth, J. Geophys. Res., 93(B11), 1371613742, 1988.

Glatzmaier, G. A. and P. H. Roberts, A three-dimensional self-consistant computer simulation of a geomagnetic field reversal, Nature, 377, 203209, 1995.

Kuang, W. and J. Bloxham, An Earth-like numerical dynamo model, Nature, 389, 371-374, 1997.

Morelli, A., A. M. Dziewonski, and J. H. Woodhouse, Anisotropy of the inner core inferred from PKIKP travel times, Geophys. Res. Lett., 13, 1545-1548, 1986.

Ritzwoller, M., G. Masters, and F. Gilbert, Observations of anomalous splitting and their interpretation in terms of aspherical structure, J. Geophys. Res., 91, 10203-10228, 1986.

Song, X., Anisotropy of the Earth's inner core, Rev. Geophys., 35(3), 297313, 1997.

Song, X.-D. and P. Richards, Seismological evidence for differential rotation of the Earth's inner-core, Nature, 382(6588), 221-224, 1996.

Souriau, A., P. Roudil, and B. Moynot, Inner core differential rotation: Facts and artifacts, Geophys. Res. Lett., 24(16), 2103-2106, 1997.

Stevenson, D. J., Limits on lateral density and velocity variations in the Earth's outer core, Geophys. J. Roy. Astron. Soc., 88, 311-319, 1987.

$\mathrm{Su}$, W.-J. and A. M. Dziewonski, Inner-core anisotropy in 3 dimensions, $J$. Geophys. Res., 100(B6), 9831-9852, 1995.

Su, W.-J., R. L. Woodward, and A. M. Dziewonski, Degree 12 model of shear velocity heterogeneity in the mantle, J. Geophys. Res., 99(B4), 6945-6980, 1994.

Su, W.-J., A. M. Dziewonski, and R. Jeanloz, Planet within a planet: Rotation of the inner core of the Earth, Science, 274, 1883-1887, 1996.

Woodhouse, J. H. and D. Giardini, Inversion for the splitting function of isolated low order normal mode multiplets, EOS Trans. AGU, 66, 300, 1985.

Woodhouse, J. H., D. Giardini, and X. D. Li, Evidence for inner core anisotropy from free oscillations, Geophys. Res. Lett., 13, 1549-1552, 1986.

D. S. Sharrock (e-mail: David.Sharrock@earth.ox.ac.uk) and J. H. Woodhouse 